\title{
A Case of Infective Endocarditis Caused by C.Hominis in a Patient with HLAB27 Aortitis
}

\author{
Tanya Suvendrini Lena, Christine De Meulemeester
}

Can. J. Neurol. Sci. 2009; 36: 385-387

A 59-year-old, right handed, Caucasian engineer presented to the emergency department 12 hours after the sudden onset of left sided weakness and numbness. The patient had been in good health until the year prior to admission. He had an episode of bilateral ankle swelling with cough, pleuritic chest pain and night sweats 12 months prior to admission. Six months later he had left ankle swelling and left foot drop, followed by right knee soreness and swelling a month later, and swelling of the left calf and right foot two months after that. He gave an eleven month history of migrating arthralgias, myalgias and periodic lower back pain. The episodes of myalgia and arthralgia typically lasted three to ten days. The patient reported some symptomatic relief with ibuprofen. He was fatigued throughout this period. There was no history of shoulder or hip pain, conjunctivitis, rash, photosensitivity, gastrointestinal or genitourinary complaints. $\mathrm{X}$-rays of the sacro-iliac joints were normal. He had developed moderate hypertension in the year prior to admission. There was no history of dental work, or dental carries. His family history was unremarkable.

Abdominal ultrasounds documented splenomegaly increasing from $13 \mathrm{~cm}$ to $18 \mathrm{~cm}$ in the year prior to admission. ESR was consistently elevated-ranging between 45 and $85 \mathrm{~mm} / \mathrm{hr}$. He had a normocytic anemia. Genetic testing revealed an HLA B27 genotype. Subsequently the patient was diagnosed with HLA B27 associated reactive arthritis.

He developed a new cardiac murmur. A transthoracic echocardiogram performed a month before admission revealed $3+$ aortic regurgitation, a mildly dilated left ventricle with normal left ventricular ejection fraction, and 1+ mitral regurgitation with a normal valve. A TTE performed four years earlier had been normal. The aortic insufficiency was deemed consistent with HLA B27 associated aortitis and a TEE was scheduled to rule out further valvular disease. The patient presented with acute stroke prior to his scheduled outpatient TEE.

On presentation he was alert and oriented. His blood pressure was $164 / 84$. He had a low grade fever. His respiratory rate was 16 and pulse was regular at 88 . He had a water hammer pulse with a JVP $5 \mathrm{~cm}$ above the sternal angle with a bifid contour. The apex was $15 \mathrm{~mm}$ in diameter and hyperdynamic. A systolic ejection click was audible at the left upper sternal border. A grade II/VI systolic crescendo-decrescendo murmur was audible at the apex and an Austin Flint rumble was present. The spleen was palpable. There was no lymphadenopathy. Skin, nails and joints were unremarkable. There was no peripheral edema. He had mild dysarthria and a left upper motor neuron facial paresis, left hemiparesis and dense left sensory loss. The left plantar response was extensor.
Admission bloodwork revealed a normal WBC with a $\mathrm{Hb}$ of 116. Electrolytes, urea, creatinine and blood glucose were normal. Urinalysis was normal. ESR was 64 and CRP was 40. An EKG met criteria for LVH. Chest x-ray was normal. The CT head showed an acute right middle cerebral artery territory infarct involving the right frontal parietal and posterior temporal regions, sparing the basal ganglia. An MRI brain showed a large acute infarct involving the right frontal parietal operculum and insula with old infarct involving the lateral portion of the right temporal pole. The MRA of the intracranial arteries showed irregularity or low flow in the posterior branch of the right middle cerebral artery. The remainder of the intracranial vessels were unremarkable. Carotid Doppler examination of the extracranial carotid arteries was normal aside from minor, nonstenosing atheromatous changes in the right mid common carotid artery.

The patient was admitted to the neurology service. Blood cultures were drawn and he was treated empirically with IV Gentamycin and Cefotaxime for presumed bacterial endocarditis. A transesophageal echocardiogram showed moderate to severe aortic insufficiency/regurgitation and a mobile mass measuring 1.12 X $0.74 \mathrm{~cm}$ on the ventricular aspect of the aortic valve cusp. The left ventricle was mildly dilated with borderline hypertrophy and a normal ejection fraction. He had 1+ mitral regurgitation with a normal valve.

Screening tests for SLE were negative. The thrombophilia screen was negative aside from an isolated elevation of anticardiolipin IgM antibodies (60 MPL U/L). This was felt to be an acute phase reactant and not causally related to the patient's stroke. The anticardiolipin IgM level was normal when rechecked seven months later. Serum protein electrophoresis was consistent with diffuse hypergammaglobulinemia. Throughout the hospital admission the patient's white blood cell count was normal. Admission blood cultures grew Cardiobacterium hominis on day nine. C hominis was subsequently isolated from three sets of blood cultures. The organism was identified using biochemical and fatty acid profiles and confirmed in triplicate at the public health laboratories in Ottawa. In vitro susceptibility testing was not performed as its interpretation is typically

From the Faculty of Medicine, Department of Neurology (TSL), University of Toronto, Toronto; Faculty of Medicine, Division of Neurology (CDM), University of Ottawa, Ontario, Canada.

Received June 5, 2008. Final Revisions Submitted January 20, 2009. Correspondence to: Tanya Suvendrini Lena, University of Toronto, Faculty of Medicine, Department of Neurology, Toronto, Ontario, Canada. 


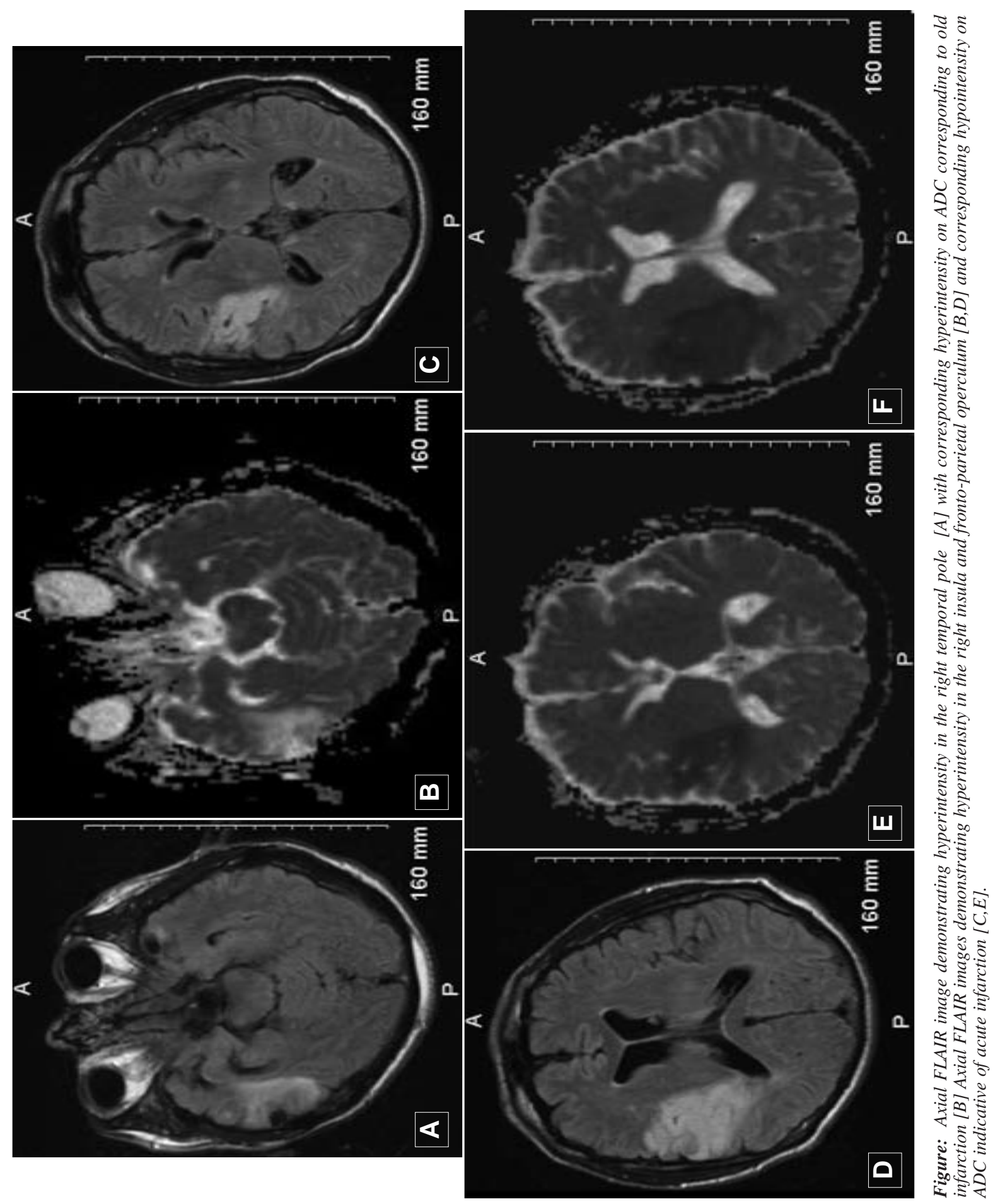


unreliable in these fastidious, slow growing organisms. Gentamycin was discontinued. Intravenous Cefotaxime was continued for a total of six weeks. Repeat blood cultures showed no growth. The patient remained clinically stable and was subsequently discharged to a stroke rehabilitation facility.

\section{Discussion}

This patient presented with Osler's triad of fever, heart murmur, and stroke leading to a clinical diagnosis of infective endocarditis. ${ }^{1}$ This case has several atypical features. First C.hominis, was isolated from blood cultures, this organism is a rare cause of bacterial endocarditis. Second, this patient presented with a very prolonged and indolent history of migratory rheumatic symptoms. Third, this case of subacute bacterial endocarditis occurred in the context of HLA B27 associated aortitis.

Cardiobacterium hominis, is a member of the HACEK group of organisms associated with culture negative endocarditis. These organisms account for approximately 3-6\% of cases of SBE diagnosed in North America ${ }^{1,2}$. C hominis is a constituent of the human oral cavity and upper respiratory tract normal flora ${ }^{2,3}$.

Clinical and microbiological characteristics of $\mathrm{C}$ hominis endocarditis were first reviewed by Wormser et al, ${ }^{4}$ and more recently by Walkty ${ }^{2}$ and Malani et al, 5 .

C Hominis infective endocarditis is remarkable for the duration and variability of symptoms that typically precede diagnosis. Infection is typically very insidious with reports of prodromes lasting for four to six months on average. The longest previously reported prodrome is eleven months ${ }^{1}$. In this case, symptoms preceded diagnosis by over a year. Patients present with non-specific symptoms such as lethargy, arthralgias and myalgias. Fever, if present, is typically low grade. Splenomegaly is the most commonly reported stigmata of endocarditis, seen in $40-59 \%$ of patients. The ESR is typically moderately elevated (91-100\% of cases). The organism has a predilection for damaged native valves or prosthetic valves. ${ }^{2-4}$.

In his early review of 32 cases Wormser reported embolic stroke in $44 \%$ of cases. Recent literature reviews report embolic stroke in $44-50 \%$ of cases $^{2,4,5}$. C hominis has also been associated with five cases of cerebral mycotic aneurysm ${ }^{6-10}$ one case of meningitis ${ }^{11}$ and one case of vertebral osteomyelitis ${ }^{12}$. Overall, the frequency and nature of neuro-logical complications of $\mathrm{C}$ hominis IE are comparable with IE resulting from other organisms ${ }^{13}$.

In this case, the history of inflammatory back pain and asymmetrical oligoarthritis in the context of HLA B27 haplotype, led to a diagnosis of reactive arthritis and HLA B27 associated aortitis. An isolated HLA B27 associated cardiac syndrome comprising cardiac conduction abnormalities, aortitis and aortic regurgitation has been defined. The link to B27 is almost as strong as with ankylosing spondylitis. Up to $10 \%$ of ankylosing spondylitis patients have aortic regurgitation, though they are often asymptomatic. HLA B27 associated cardiac lesions may also be found in the absence of other rheumatological complaints and may represent a forme fruste of ankylosing spondylitis ${ }^{14-17}$.

There is no known association between HLA B27 and susceptibility to infective endocarditis ${ }^{18}$. This case raises the intriguing possibility that an HLA B27 associated aortitis may have served as a nidus for $C$ hominis infective endocarditis. The syndrome causes fibrotic thickening and downward retraction of the bases of the aortic cusps and inward rolling of the edges or margins of the cusps. These changes may make the aortic valve in HLA B27 aortitis vulnerable to seeding by a variety of organisms, constituting a risk factor for infective endocarditis and stroke.

\section{ACronym Definitions}

TTE-transthoracic echocardiogram; TEE-transesophageal echocardiogram; JVP-jugular venous pressure; ESR-erythrocyte sedimentation rate; CRP-c reactive protein; LVH-left ventricular hypertrophy; MRA-magnetic resonance angiography; MRImagnetic resonance imaging; SLE-systemic lupus erythematosis; HLA-human leukcocyte antigen

\section{REFERENCES}

1. Geraci JE, Griepp PR, Wilkowske CJ, Wilson WR, Washington JA. Cardiobacterium hominis endocarditis: four cases with clinical and laboratory observations. Mayo Clin Proc. 1978; 53:49-53.

2. Walkty A.Cardiobacterium hominis endocarditis: A case report and review of the literature. Can J Infect Dis Med Microbiol. 2005; 16(5):293-7.

3. Slotnick IJ, Mertz AJ, Dougherty M. Fluorescent antibody detection of human occurrence of an unclassified bacterial group causing endocarditis. J Infect Dis. 1964; 114:503-5.

4. Wormser GP, Bottone EJ. Cardiobacterium hominis: review of microbiologic and clinical features. Rev Infect Dis. 1983; 5(4): 680-91.

5. Malani AN, Aronoff DM, Bradley SF, Kauffman CA. Cardiobacterium hominis endocarditis: two cases and a review of the literature. Eur J Clin Microbiol Infect Dis. 2006; 25(9): 587-95.

6. Perdue GD, Dornery ER, Ferrier F. Embolomycotic aneurysm associated with bacterial endocarditis due to cardiobacterium hominis. Am Surg.1968; 34(12):901-4.

7. Lynfield, J Nelson PK. Mycotic Aneurysm. NEJM. 2002; 347 (25):2030.

8. Lin BH, Vieco PT. Intracranial mycotic aneurysm in a patient with endocarditis caused by Cardiobacterium hominis. Can Assoc Radiol J. 1995; 46(1):40-2.

9. Laguna J, Derby BM, Chase R. Cardiobacterium hominis endocarditis with cerebral mycotic aneurysm. Arch Neurol. 1975; 32(9)638-9.

10. Han XY, Meltzer MC, Woods JT, Fainstein V. Endocarditis with ruptured cerebral aneurysm caused by Cardiobacterium valvarum sp. nov. J Clin Microbiol. 2004;42(4):1590-5.

11. Francioli PB, Roussianos D, Glauser MP. Cardiobacterium hominis endocarditis manifesting as bacterial meningitis. Arch Intern Med. 1983; 143(7):1483-4.

12. Nurnberger M, Treadwell T, Lin B, Weintraub A. Pacemaker lead infection and vertebral osteomyelitis presumed due to Cardiobacterium hominis. Clin Infect Dis, 1998; 27(4):890-1.

13. Prabhakaran S. Neurologic complications of endocarditis. Continuum. 2008; 14(1):53-73.

14. Bergfeldt L. HLA B-27 Associated cardiac disease. Ann Intern Med. 1997; 127 (8 Pt 1): 621-9.

15. Bergfeldt L, Insulander P, Lindblom D, Moller E, Edhag O. HLAB27: an important genetic risk factor for lone aortic regurgitation and severe conduction system abnormalities. Am J Med. 1988; 85(1):12-8

16. Mader R. Atypical clinical presentation of ankylosing spondylitis. Semin Arthritis Rheum. 1999; 29(3):191-6.

17. Lautermann D, Braun J. Ankylosing spondylitis--cardiac manifestations. Clin Exp Rheumatol. 2002; 20 (6 Suppl 28): S11-5.

18. Sheehan NJ. The ramifications of HLA B27. J R Soc Med. 2004; 97:10-14. 